$5-1-2008$

\title{
Tasting Phenylthiocarbamide (PTC): A New Integrative Genetics Lab with an Old Flavor
}

\author{
Robert B. Merritt \\ Smith College, rmerritt@smith.edu \\ Lou Ann Bierwert \\ Smith College, Ibierwer@smith.edu \\ Barton Slatko \\ New England Biolabs \\ Michael P. Weiner \\ Rothberg Institute for Childhood Diseases \\ Jessica Ingram \\ New England Biolabs
}

See next page for additional authors

Follow this and additional works at: https://scholarworks.smith.edu/bio_facpubs

Part of the Biology Commons

\section{Recommended Citation}

Merritt, Robert B.; Bierwert, Lou Ann; Slatko, Barton; Weiner, Michael P.; Ingram, Jessica; Sciarra, Kristianna; and Weiner, Evan, "Tasting Phenylthiocarbamide (PTC): A New Integrative Genetics Lab with an Old Flavor" (2008). Biological Sciences: Faculty Publications, Smith College, Northampton, MA. https://scholarworks.smith.edu/bio_facpubs/66

This Article has been accepted for inclusion in Biological Sciences: Faculty Publications by an authorized administrator of Smith ScholarWorks. For more information, please contact scholarworks@smith.edu 


\section{Authors}

Robert B. Merritt, Lou Ann Bierwert, Barton Slatko, Michael P. Weiner, Jessica Ingram, Kristianna Sciarra, and Evan Weiner 


\section{BioOne COMPLETE}

\section{Tasting Phenylthiocarbamide (PTC): A New Integrative Genetics Lab with an Old Flavor}

Authors: Merritt, Robert B., Bierwert, Lou Ann, Slatko, Barton, Weiner, Michael P., Ingram, Jessica, et. al.

Source: The American Biology Teacher, 70(5)

Published By: National Association of Biology Teachers

URL: https://doi.org/10.1662/0002-7685(2008)70[23:TPPANI]2.0.CO;2

BioOne Complete (complete.BioOne.org) is a full-text database of 200 subscribed and open-access titles in the biological, ecological, and environmental sciences published by nonprofit societies, associations, museums, institutions, and presses.

Your use of this PDF, the BioOne Complete website, and all posted and associated content indicates your acceptance of BioOne's Terms of Use, available at www.bioone.org/terms-of-use.

Usage of BioOne Complete content is strictly limited to personal, educational, and non - commercial use. Commercial inquiries or rights and permissions requests should be directed to the individual publisher as copyright holder.

BioOne sees sustainable scholarly publishing as an inherently collaborative enterprise connecting authors, nonprofit publishers, academic institutions, research libraries, and research funders in the common goal of maximizing access to critical research. 
monohybrid and dihybrid cross ratios, results from the application of two basic rules of probability: the rule of products and the rule of sums. The rule of products states that the probability of two independent events occurring together is the product of their separate probabilities - if the probability of a $T$ gamete is $p$, the probability of a TT zygote will be $\mathrm{p}^{2}$. The rule of sums states that if there are two or more mutually exclusive ways of achieving the same outcome, the probability of that outcome is the sum of the probabilities of the separate ways of achieving it - a Tt zygote may result from either a $T$ egg or a $t$ egg, hence the probability to a Tt zygote is 2pq. As an aside here, monohybrid Punnett squares are a valuable heuristic tool because we are such a visual species, but they are best used to teach the rule of products, the rule of sums, and conditional probability (see below) rather than simply to visualize monohybrid ratios. Students should be discouraged from constructing more complicated Punnett squares (dihybrid, trihybrid, etc.) but encouraged to derive these ratios from a simple application of the rule of products:

$f(A-B-)=(3 / 4)(3 / 4)=9 / 16, f(A-b b)=(3 / 4)(1 / 4)=3 / 16$, $f(a a B-)=(1 / 4)(3 / 4)=3 / 16, f(a a b b)=(1 / 4)(1 / 4)=1 / 16$.

\section{Determining PTC Genotypes}

Nontasters (tt) know their genotypes since they have to be homozygous recessive to express their phenotype. Such is not the case for tasters ( $T$-), and the tasters in the class will be curious about whether they are homozygotes (TT) or heterozygotes $(T t)$. Until recently there have been two approaches to answering this question: one that involves using the class allele frequencies to calculate the conditional probability that a taster in the class is either homozygous or heterozygous, and another that adds pedigree analysis to the determination of genotype probabilities. Conditional probabilities are calculated by dividing the probability of the outcome of interest by the probability of the condition. In our hypothetical class above, a taster could calculate the probability that she was a homozygote by dividing the frequency of taster homozygotes (f[TT]) by the frequency of tasters (f[T-]) or $(0.45)^{2} / 0.70$. Hence the probability that a taster is homozygous is 0.29 and the probability a taster is heterozygous is 0.71 . Here again is an opportunity to refer back to Mendel's monohybrid cross results where he expected $1 / 3$ of his $\mathrm{F}_{2}$ dominants to breed true since $(1 / 4) /(3 / 4)=1 / 3$.

Pedigree data can provide additional insight into the genotype of taster students. If a taster student has a nontaster parent, she must be a heterozygote (probability of $T T=0$ ). If the taster student has two taster parents and at least one nontaster sibling, the probability of homozygosity is $1 / 3$ (again, a conditional probability). If the taster student has two taster parents, no nontaster siblings, and no information on grandparents, the probability of homozygosity can be calculated using the conditional probabilities that a taster is homozygous (0.29) or heterozygous (0.71) estimated above using the class population data. The probability that the taster student is homozygous in this case would be the sum of probability of TTxTT matings $\left(0.29^{2}\right)$ times 1.0 (probability of TT progeny from this cross) or 0.084 plus the probability of TTxTt matings $(2 \times 0.29 \times 0.71)$ times $1 / 2$ (the probability of TT progeny from this cross) or 0.206 plus the probability of TtxTt matings $\left(0.71^{2}\right)$ times $1 / 3$ (the probability of $T T$ progeny among $\mathrm{T}$ - progeny from this cross) or 0.168 . Therefore, the probability that such a taster student is homozygous (given the original class data) is 0.46 . While the PTC polymorphism provides an interesting opportunity for students to employ their expertise in pedigree analysis, it should be remembered that the actual collection of pedigree data carries the risk of revealing sensitive information. Even with pedigree data, over half of the taster students in the above class would be uncertain of their PTC genotype - they would be left with a probability of homozygosity verses heterozygosity. With the identification and sequencing of the PTC gene, that uncertainty can now be eliminated.

It is now possible to use cleaved amplified polymorphic sequence (CAPS) analysis to determine student genotypes for the PTC gene on the long arm of chromosome 7 (7q35-q36). The sequence of this gene, which is primarily responsible for variation in ability to taste PTC, is shown in Figure 1 (NCBI data bank). Two common alleles or haplotypes (and in some cases, a few rarer alleles) are found in most human populations. The taster and nontaster haplotypes differ by three single nucleotide polymorphisms (SNPs) shown in bold red in Figure 1. The sequence shown in the figure is that of the common nontaster allele with $\mathrm{G}$ at nucleotide position 145 (G145), T at position 785 (T785) and A at position 886 (A886). This nontaster allele produces a polypeptide with alanine at amino acid position 49, valine at position 262, and isoleucine at position 296, and is referred to as the AVI allele. The common taster allele has C145, C785, and G886, produces a polypeptide with 49proline, 262alanine, and 296valine, and is referred to as the PAV allele. The SNP at position 785 is of particular interest in genotyping since the taster sequence in this region forms an Fnu4Hl restriction site (see Figure 1) while replacing C785 with T785 in the nontaster allele eliminates this restriction site. There are two other Fnu4Hl restriction sites (shown in dark red in Figure 1) within the PTC gene, but primers (shown in green and blue in Figure 1) have been specifically designed to bind to regions flanking the one polymorphic Fnu4Hl site while excluding the other two. Using these primers in a polymerase chain reaction (PCR) generates 303 base pair fragments. Each student can obtain these fragments by amplifying isolated cheek cell DNA. PCR amplification can then be followed by a restriction digest using Fnu4H1. Following electrophoretic separation of the digestion products, nontaster homozygotes will still see only the 303 base pair fragment on the gel, while taster homozygotes will see two shorter fragments (239bp and 65bp) and taster heterozygotes will see all three fragment lengths. A typical gel is shown in Figure 2. This CAPS analysis allows students to apply their knowledge of DNA structure and replication, the genetic code, PCR, restriction endonucleases, and electrophoresis. A step-bystep protocol for the RFLP analysis is provided at the end of this article.

\section{Molecular Evolution, Balancing Selection \& the PTC Gene}

Finally, a review of recent literature on the PTC gene can provide students with insight into how evolutionary relationships are unraveled. Kim et al. (2003) established that the PAV (taster) allele is the ancestral state in humans because these same three amino acids (proline, alanine, and valine) occur at the same positions in the PTC gene product from chimpanzee, lowland gorilla, orangutan, an old world monkey (crab-eating macaque), and a new world monkey (black-handed spider monkey). Two observations have been used to argue that the PTC alleles are maintained in human populations by balancing selection: the widespread occurrence of the polymorphism and the fact that chimpanzees show a similar phenotypic variation. Fisher et al., (1939) proposed that the origin of the two common alleles occurred prior to the separation of the human and 
Figure 1. The Human PTC Gene. The gene that is primarily responsible for human PTC taste sensitivity is located on the long arm of chromosome 7. The sequence of the nontaster allele is shown below with attention drawn to common single nucleotide polymorphism sites (SNPs), Fnu4H1 restriction endonuclease sites, primer sites for gene amplification by PCR. The Figure also indicates the amino acid substitutions corresponding to the SNPs, the restriction digest fragment lengths obtained in RFLP analysis, and the amino acid sequence for the nontaster gene product. The amino acid sequence also indicates the initiation site for translation of the chimpanzee nontaster allele (light blue M). Note that the amino acid sequence given is still for the human nontaster allele, not the chimpanzee allele.

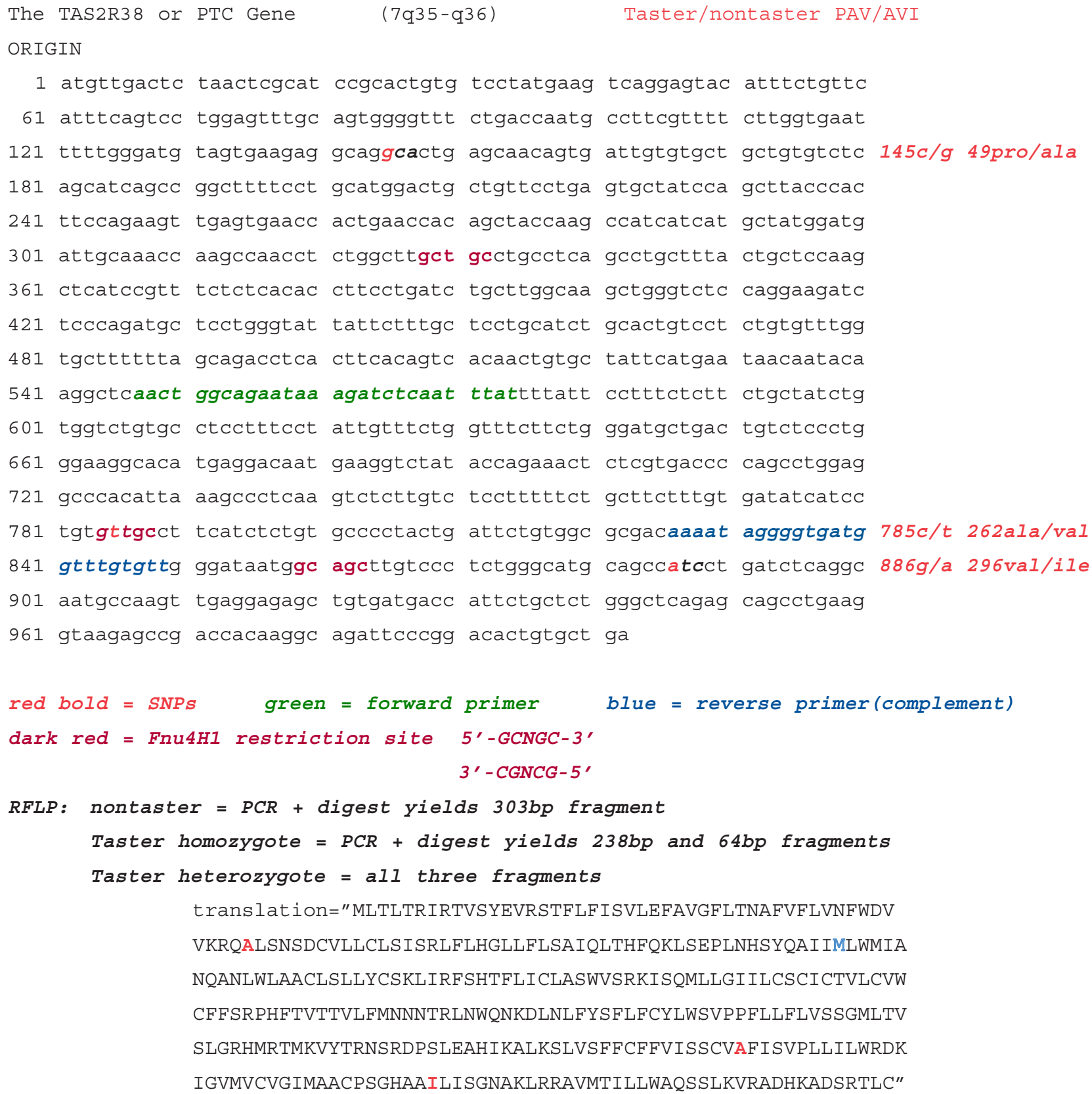

chimpanzee lineages and has been maintained in both species by balancing selection. However, while Wooding et al. (2006) demonstrated that indeed the same gene is primarily responsible for variation in PTC taste sensitivity in both species, the chimpanzee nontaster allele results from a mutation in the start codon (ATG to $A G G$ ) so that translation is initiated with the methionine at what is the 97th amino acid (see light blue $\mathrm{M}$ in translation sequence in Figure 1) in the taster polypeptide chain. These same authors also provide evidence that this truncated chimpanzee gene product is nonfunctional. Wooding et al. (2006) conclude that the nontaster allele in chimpanzees may be selectively neutral and that nontaster alleles in humans and chimpanzees are clearly of independent origin. Although Fisher may not have been correct about the origin of the PTC alleles, he was almost 
certainly correct about the role of selection in maintaining the alleles in human populations (Wooding et al., 2004).

It seems likely that these bitter taste receptors influence food preferences that are somehow related to reproductive success. Finally, while the PAV and AVI alleles account for more than $90 \%$ of the variation in the PTC gene, a total of five additional haplotypes have been identified, with allelic diversity greatest in sub-Saharan Africa. The identification and sequencing of the PTC gene should stimulate new interest in this old favorite of both anthropologists and geneticists, and clearly provides an opportunity for an unusually integrative laboratory experience for students in advanced biology/genetics courses.

\section{Protocol for CAPS Analysis*}

\section{Cheek Cell DNA Extraction}

1. Scrape inside of cheek with a sterile plastic loop (Fisher Scientific).

2. Twirl loop in 200ul of 5\% Chelex buffer (Carolina Biological) in a $1.5 \mathrm{ml}$ screw-cap microfuge tube.

3. Add 2 ul of $10 \mathrm{mg} / \mathrm{ml}$ Proteinase K (Fisher Scientific) to the tube and incubate for 15-30 minutes at $56^{\circ}$ C.

4. Vortex for 10 seconds.

5. Microfuge at maximum speed for 20 seconds.

6. Boil for eight minutes.

7. Vortex for ten seconds.

8. Microfuge at maximum speed for two to three minutes.

\section{PCR Reaction}

Components for the PCR reaction (AmpliTaq Gold DNA Polymerase with 10X PCR buffer, GeneAmp dNTP Blend, Applied Biosystems) are thawed, gently mixed, briefly microfuged, and stored on ice. A master mix is made as follows. For each sample to be amplified, add:

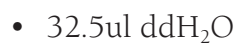

- $0.5 \mathrm{ul} 10 \mathrm{mM}$ dNTPs

- 3 ul $25 \mathrm{mM} \mathrm{MgCl}$

- 5ul 10X PCR Gold Buffer

- lul Forward Primer (10uM) 5'AACTGGCAGAATAAAGATCTCAATTTAT3'

- lul Reverse Primer (10uM) 5'AACACAAACCATCACCCCTATTTT3'

- 0.5ul AmpliTaq Gold Polymerase

This master mix should be gently mixed by flicking with index finger, quickly microfuged, and dispensed in $43.5 \mathrm{ul}$ aliquots into PCR tubes. Each student should add 6.5ul of the supernatant from his/her DNA extraction tube to individual PCR tube of master mix. The PCR tubes containing the 50ul reaction mixtures will be placed in a PCR machine (or hand cycled if a
Figure 2. Cleaved amplified polymorphic sequence (CAPS) Gel. The samples on the gel read from left to right as follows: slots 1,4,6 and 9 - taster heterozygotes; slots 2,3, and 8 - nontaster homozygotes; slot 5 - taster homozygote; slot 7 - blank; slot 10 - 100 base pair ladder. Nontasters have only the uncut 303bp fragment, taster homozygotes the 238 and 64bp fragments. Taster heterozygotes have all three fragments. The $64 \mathrm{bp}$ fragments are faint as a result of diffusion.

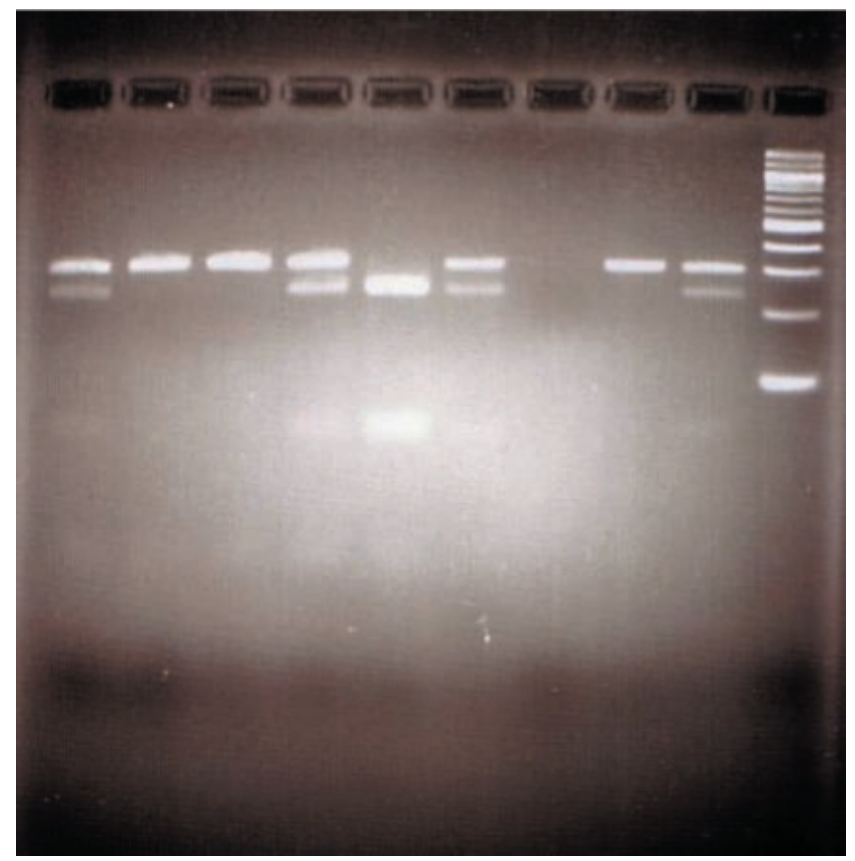

PCR machine is not available) and run as follows:

- 1 cycle of

$95^{\circ} \mathrm{C}$ for 10 minutes

$55^{\circ} \mathrm{C}$ for five minutes

- 40 cycles of

$72^{\circ} \mathrm{C}$ for 90 seconds

$95^{\circ} \mathrm{C}$ for 45 seconds

$55^{\circ} \mathrm{C}$ for 45 seconds

- 1 cycle of

$72^{\circ} \mathrm{C}$ for 10 minutes

$4^{\circ} \mathrm{C}$ until collected

After cycling, the reactions can be stored at $-20^{\circ} \mathrm{C}$ or immediately used for restriction digestion. No post-PCR clean-up is necessary.

\section{Fnu4H1 Digestion}

A restriction digest master mix is made as follows (per student):

- $6 u l d d H_{2} \mathrm{O}$

* Note: While we have had excellent results using this protocol, teachers may prefer the convenience of the Human PTC DNA Haplotyping Kit (AT) from Carolina Biological Supply Company (FR-21-1376). The Carolina CAPS assay involves a different region of the PTC taste gene and uses PTC haplotyping to introduce students to bioinformatics rather than taking the more integrative approach suggested here. 
- 3ul 10X NEB RE Buffer \#4 $(\mathrm{NEB}=\mathrm{New}$ England Biolabs, Inc.)

- lul Fnu4H1 Restriction Endonuclease (NEB)

This restriction digest master mix should be mixed gently, quickly microfuged, and dispensed as 10ul aliquots into $1.5 \mathrm{ml}$ microfuge tubes. Each student then adds 20ul of his/ her PCR product to an individual tube of 10ul restriction digest master mix and places the tube in a $37^{\circ} \mathrm{C}$ water bath overnight.

\section{Electrophoresis of Restriction Enzyme Digest}

1. Make a 3\% agarose gel (3 grams SeaKem LE Agarose from BioWhittaker Molecular Applications per 100ml) with LB buffer from Faster Better Media LLC. Allow the gel to cool and remove the combs. (Note: For sharper bands, incubate gel in stain for one hour prior to loading samples.)

2. Each student will add 15ul of digest (half the digest tube contents) to $3 \mathrm{ul}$ of $5 \mathrm{X}$ Blue Juice dye (American Bioanalytical) and load entire 18ul into a well on the gel. (Note: To emphasize the difference between amplification and digestion, students may want to load the remainder of their undigested PCR product on the gel next to their digest.)

3. Electrophorese the gel at 300 volts for 30 minutes.

4. If the gel was not stained prior to loading samples, stain with SYBR Safe (Invitrogen) for 30 minutes.

5. Photograph: for appropriate filters see - http://probes. invitrogen.com/products/sybrsafe/filters.html.

\section{Manual PCR Using Inexpensive Household Materials}

Through experimentation, we have determined that manual PCR using kitchen appliances can be used for the amplification of buccal swab DNA. Although manual PCR is a time-consuming process, it is easy and can replace expensive lab equipment in situations where budgets may be limited. In manual PCR, the reaction-containing tubes are moved manually between aluminum pots containing a suitable heat-retaining substrate (i.e., water or, preferably, sand). The cost for three hot plates, three pots, three thermometers, and a kitchen timer is approximately $\$ 50$. Although it increases the overall cost slightly, digital thermometers are recommended and can be pur- chased at most electronics or pet stores. Fine-grade sand (of the kind used in children's playgrounds) is heated to the three temperatures used in a PCR (Figure 3). In setting up the temperatures, we found that it was important to stir the sand thoroughly to obtain even heating. As a thermal sink, sand is more useful than water because it retains heat for a longer period of time. However, a disadvantage to using sand, rather than water, is that if the sand is overheated (which is easy to do since sand heats rather rapidly), it is difficult to bring the temperature down in a timely fashion. The temperature of the hot plates can usually be stabilized in approximately 15 minutes. Allow the temperatures to remain stable for approximately 20 minutes. The reactions must be overlaid with mineral oil (to prevent condensation of the reactionmix on the tube top) and tightly capped. Times and temperatures remain consistent with those previously described for automated thermal cycle-based PCR, but the number of cycles may need to be increased. A sheet of paper (see Figure 3) that can be checked-off is used to track the number of cycles (note that temperatures on sample sheet differ from those suggested for PTC-PCR).

\section{A Special Note on PTC Taste Paper Safety}

In Investigating Safety: A Guide for High School Teachers, Texley et al. (2004) characterize the way in which biology teachers have used PTC taste papers as "inaccurate, dangerous, and downright bad science." The 75 years of research on PTC taste and our discussion in this article should render any response to the "inaccurate" and "downright bad science" characterizations unnecessary. However, we worry that the "dangerous" characterization in what we understand is a widely-used reference may raise concerns for some teachers. There is no question that PTC is toxic (LD50 in rat is $3 \mathrm{mg} / \mathrm{kg}$, in mouse $10 \mathrm{mg} / \mathrm{kg}$, and in rabbit $40 \mathrm{mg} / \mathrm{kg}$ ), but so is table salt (acute toxicity in humans at $500-1000 \mathrm{mg} / \mathrm{kg}$ ). The issue is how much PTC is on a taste paper. Texley et al. indicate that "a single strip contains about $0.3 \mathrm{mg}$ " but the two suppliers we checked with indicate that a taste paper contains either $0.007 \mathrm{mg}$ (Carolina Biological Supply Company) or $0.005-0.007 \mathrm{mg}$ (ScienceStuff). Assuming a linear dose response curve, we calculate that the $230 \mathrm{mg}$ of $\mathrm{NaCl}$ in a vending machine bag of potato chips is about 100 times more toxic than the $0.007 \mathrm{mg}$ of PTC in a taste paper. We do not believe there is any reason for teachers to be concerned about the toxicity of PTC taste papers. 


\section{Acknowledgments}

This work was supported in part by a Blakeslee Genetics Research Grant to Smith College. Figure 2 is courtesy of the ninth grade biology classes at the Park Tudor School in Indianapolis, Indiana. High school students in the Smith College Summer Science and Engineering Program contributed to the refinement of this laboratory exercise.

\section{References}

Fisher, R.A., Ford, E.B. \& Huxley, J. (1939). Taste-testing the anthropoid apes. Nature, 144, 750.

Guo, S. \& Reed, D.R. (2001). The genetics of phenylthiocarbamide perception. Annals of Human Biology, 28, 111-142.

Kim, U., Jorgenson, E., Coon, H., Leppart, M., Risch, N. \& Drayna, D. (2003). Positional cloning of the human quantitative trait locus underlying taste sensitivity to phenylthiocarbamide. Science, 299, 1221-1225.

Kim, U., Wooding, S., Ricci, D., Jorde, L.B. \& Drayna, D. (2005). Worldwide haplotype diversity and coding sequence variation at human taste receptor loci. Human Mutation, 26(3), 199-204.

Textley, J.V., Kwan, T. \& Summers, J. (2004). Investigating Safety: A Guide for High School Teachers. Arlington, VA: NSTA Press.

Wooding, S. (2006) Phenylthiocarbamide: A 75 year adventure in genetics and natural selection. Genetics, 172(4), 2015-2023.

Wooding, S., Kim, U., Bamshad, M.J., Larsen, J., Jorde, L.B. \& Drayna, D. (2004). Natural selection and molecular evolution in PTC, a bitter-taste receptor gene. American Journal of Human Genetics, 74, 637-646.

Wooding, S., Bufe, B., Grassi, C., Howard, M.T., Stone, A.C., Vazquez, M., Dunn, D.M., Meyerhof, W., Weiss, R.B. \& Bamshad, M.J. (2006). Independent evolution of bitter-taste sensitivity in humans and chimpanzees. Nature, 440, 930-934. 